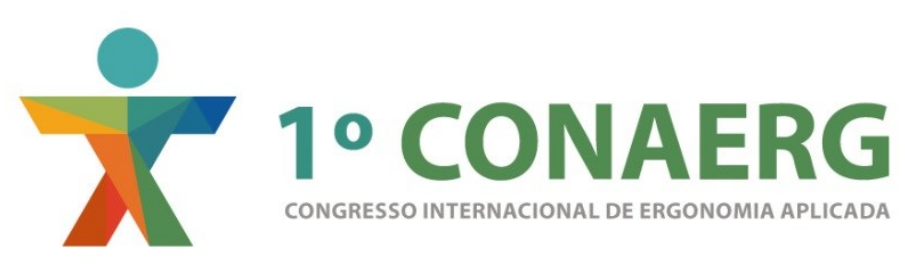

\title{
PERCEPÇÃO DAS CONDIÇÕES DE TRABALHO E SAÚDE PELOS AGENTES PENITENCIÁRIOS DO PRESIDIO MASCULINO DE FLORIANÓPOLIS/SC
}

\author{
Fabíola Reinert (1); \\ Lizandra Garcia Lupi Vergara (2); \\ Leila Amaral Gontijo (3); \\ (1) Universidade Federal de Santa Catarina, Doutoranda \\ e-mail:fabiola.reinert@gmail.com \\ (2) Universidade Federal de Santa Catarina, Dr. \\ e-mail:I.vergara@ufsc.br \\ (3) Universidade Federal de Santa Catarina, Dr. \\ e-mail:leila.gontijo@ufsc.br
}

\begin{abstract}
RESUMO
Partindo-se das hipóteses de que a saúde do trabalhador é amplamente influenciada pela sua condição de trabalho e que o trabalho em presídios contribui para a incidência de problemas de saúde, este estudo de caso investiga a real percepção dos agentes penitenciários do Presídio Masculino de Florianópolis/SC sobre seu trabalho e saúde, a partir da técnica de análise do conteúdo. O estudo desenvolvido enfatiza que o tipo de trabalho e seu conteúdo elevam o nível de stress dos trabalhadores analisados, sendo que a falta de segurança gera medo e desconfiança, revelando problemas na organização e no processo de trabalho.
\end{abstract}

\begin{abstract}
Starting from the hypothesis that workers' health is largely influenced by its working condition and that the work in prisons contributes to the incidence of health problems, this case study investigates the real perception of correctional officers of Male Prison from Florianopolis/SC about their work and health, using the content analysis technique, with guided interview. The study developed emphasizes that the type of work and its contents raise the stress level of the analyzed workers, and the lack of security creates fear and mistrust, revealing problems in the organization and work process.
\end{abstract}

\section{INTRODUÇÃO}

Diversos autores documentaram os efeitos das condições de trabalho na saúde dos trabalhadores (CHENG et al., 2000; ISHIZAKI et al., 2006; CASSITTO et al., 2003), e descreveram a associação entre um ambiente de trabalho severo e um vasta gama de doenças, incluindo principalmente doenças mentais (STANSFELD et al., 2006; BABAZONO et al., 2005; HIGASHIGUCHI et al., 2002). 
Segundo Tsutsumi et al. (2002) e Johnson et al. (2005), a exposição a fatores de risco no ambiente de trabalho varia de acordo com o tipo de ocupação e o cargo. Em servidores penitenciários foram observados altos níveis de fatores de risco, especialmente nos indivíduos em contato direto com os detentos, que trabalham em um ambiente caracterizado por um alto nível de exigências psicológicas (JOHNSON et al., 2005; GHADDAR et al., 2008). De acordo com a Organização Internacional do Trabalho (OIT, 2014), a profissão é a $2^{a}$ mais perigosa do mundo, sendo um dos poucos cargos onde incidem periculosidade e insalubridade ao mesmo tempo.

Dejours (1992) afirma que as condições e organização do trabalho têm estreita relação com as mais diversas doenças. O trabalho pode ser fonte de sofrimento e doença, e é preciso compreender como os trabalhadores mantêm seu equilíbrio psíquico, mesmo quando submetidos a condições de trabalho desestruturantes. $\mathrm{O}$ ambiente de trabalho em unidades prisionais é diferente de qualquer outro, com a possível exceção do ambiente existente em instituições psiquiátricas e outras instituições de confinamento (BOURBONNAIS et al., 2007). Segundo Vasconcelos (2000), o trabalho em unidades prisionais transforma os trabalhadores, por ser permeado pelo fenômeno da violência.

A precariedade das unidades prisionais de Santa Catarina foi estudada (DAMAS, 2012) relacionando a superlotação, infra-estrutura precária e déficit em recursos humanos com problemas de saúde como: HIV, tuberculose, doenças respiratórias, dermatoses e transtornos mentais. Em análise das condições de trabalho do assistente social no Presídio Feminino de Florianópolis/SC (REINERT et al., 2014) foi percebido a falta de recursos, higiene e ventilação no presídio, além da sobrecarga e elevado nível de stress no trabalhador.

Um estudo sobre a saúde em prisões do Rio de Janeiro (DIUANA et al., 2008), a ameaça à integridade pessoal do agente penitenciário aparece como inerente ao trabalho, além de tensão emocional, manifestações psicossomáticas e estresse. Pesquisas feitas com agentes penitenciários do Rio de Janeiro (VASCONCELOS, 2000), de Salvador (FERNANDES et al., 2002) e de São Paulo (RUMIN, 2006) identificaram riscos biológicos de contaminação por tuberculose e hepatite, em função das péssimas condições de trabalho. Rumin (2006) ainda identificou alto risco de violência.

Estudos realizados por Santos (2010) sobre as influências negativas do trabalho em penitenciárias do Rio Grande do Sul e do Rio Grande do Norte, sob a ótica dos próprios servidores penitenciários, mostraram medo e insatisfação constantes. Santos et al. (2010) sugere que os agentes penitenciários no Hospital de Custódia e Tratamento Psiquiátrico André Teixeira Lima (SP) estão expostos a situações de altas demandas psicológicas no trabalho, com $83,3 \%$ preenchendo critérios para a presença de Transtornos Mentais Comuns (TMC) e o trabalho de Gomes (2009) com professores atuantes em complexos penitenciários brasileiros, afirma que $12,5 \%$ apresentam distúrbios psíquicos, considerados como indicadores de evidências de sofrimento mental.

Comparando os agentes penitenciários a outras profissões, foi encontrado grande prevalência de ansiedade, hipertensão, doenças psicossomáticas, e reações comportamentais (p.ex. abuso de drogas) (JOHNSON et al., 2005; BOURBONNAIS et al., 2007), além de alta freqüência de reações relacionadas ao stress nesse grupo, como absenteísmo e doenças psicológicas (CAMP et al., 2006; GOLDBERG et al, 1996, TARTAGLINI; SAFRAN, 1997). Também, uma baixa expectativa de vida foi reportada para agentes penitenciários comparados a outras profissões, e isso foi atribuído ao stress ocupacional (CHEEK, 1984; WOODRUFF, 1993).

A gravidade dos efeitos físicos e psicológicos do estresse sobre o agente penitenciário (doença cardíaca, hipertensão, úlceras pépticas, alcoolismo, depressão, suicídio, ansiedade, bem como outros transtornos mentais), como posta por Anson et al. (1997) e DeCarlo e GruenFeld (1989), tem sérias implicações para a gestão da saúde mental neste tipo de organização. 
Uma Escala de Estresse no Trabalho foi aplicada em 119 agentes penitenciários da Turquia (SENOL-DURAK, 2006), obtendo como resultado sintomas de depressão, ansiedade, desesperança e falta de apoio social percebido nos sujeitos pesquisados. Tartaglini e Safran (1997), referem-se aos agentes penitenciários como profissionais submetidos a um alto risco para a doença relatada como estresse debilitante. Estes autores encontraram prevalências de ansiedade, distúrbios de comportamento e abuso de álcool mais altos entre os agentes do que na população em geral. Relataram entre esses trabalhadores, uma prevalência de distúrbios emocionais de $18,6 \%$, abuso de álcool de $4,5 \%$ e distúrbios da ansiedade de $7,9 \%$.

Bourbonnais et al. (2007) obtiveram como resultado em um estudo realizado no sistema prisional de Quebec com 1881 agentes prisionais, uma associação entre o sofrimento psíquico e altas demandas psicológicas, baixo poder de decisão, uma combinação dessas duas restrições e baixo apoio social no trabalho. Em estudo com 164 agentes penitenciários do Centro Penitenciário Espanhol concluiu que esse trabalho gera altas demandas psicológicas, baixa auto-estima, baixo controle, baixo apoio social, alta exposição e insegurança no trabalho (GHADDAR et al., 2008).

Agentes penitenciários que trabalham em ambiente carcerário fechado, severo e coercitivo, tendo múltiplas responsabilidades para a correção, a educação e a gestão dos prisioneiros e à manutenção da segurança e da ordem dentro das prisões, são propensos a sofrer vários problemas mentais, especialmente depressão (GOLDBERG et al., 1996; BOURBONNAIS et al., 2007; OBIDOA et al., 2011). Em um estudo francês, a proporção global de agentes penitenciários com sintomas depressivos foi de 24\% (GOLDBERG et al., 1996) .

Aproximadamente um terço dos agentes penitenciários empregados em duas prisões do estado no nordeste dos EUA informou sofrimento psíquico grave (OBIDOA et al., 2011). Pesquisas com 1900 agentes prisionais de quatro prisões masculinas da China mostrou altos níveis de sintomas depressivos, em torno de 60\% (LIU et al., 2013; SUI et al., 2014). Além disso, segundo Armstrong e Griffin (2004), o ambiente prisional é o único em que os trabalhadores percebem uma ameaça constante de perigo daqueles que supervisionam.

Neste contexto, partindo-se das hipóteses de que a saúde do trabalhador é amplamente influenciada pela sua condição de trabalho e que o trabalho em presídios contribui para a incidência de problemas de saúde, pretende-se verificar a real percepção dos agentes penitenciários sobre seu trabalho e saúde.

O futuro do trabalho, com a evolução da sociedade, caminha para funções e profissões com cada vez mais exigências mentais, assim como o futuro da ergonomia, que tem passado a se preocupar em compreender os fatores psicológicos e psicossociais, além dos fatores físicos e biomecânicos, sendo de grande valia entender como as condições de trabalho influenciam na saúde dos trabalhadores.

\section{METODOLOGIA}

Esta pesquisa tem caráter exploratório, com o objetivo de detectar, compreender e interpretar o fenômeno investigado (GIL, 1996). A pesquisa exploratória, segundo Trivinõs (2006), permite ao pesquisador aprofundar suas análises nos limites de uma realidade específica.

Após o levantamento bibliográfico sobre o assunto, partiu-se para uma pesquisa de abordagem qualitativa, através de um estudo de caso, que se caracteriza como um tipo de pesquisa cujo objeto é uma unidade que se analisa aprofundadamente (TRIVINÕS, 2006). Sendo assim, foi investigada a saúde dos agentes penitenciários do Presídio Masculino de Florianópolis, a partir da técnica de análise do conteúdo, utilizando entrevista guiada, para 
uma análise comparativa através da construção de tipologias, problemáticas e análises temáticas.

Segundo Bardin (2009), a análise de conteúdo, enquanto método, torna-se um conjunto de técnicas de análise das comunicações que utiliza procedimentos sistemáticos e objetivos de descrição do conteúdo das mensagens.

Para a análise de conteúdo foram realizadas três etapas: pré-análise, exploração do material e tratamento e interpretação dos resultados. Segundo Bardin (2009) a fase de préanálise visa sistematizar as ideias para elaborar um esquema preciso de desenvolvimento do trabalho. Nesta etapa são feitas a escolha do material, formulação das hipóteses e dos objetivos, e elaboração de indicadores da interpretação final. $\mathrm{Na}$ fase de exploração do material, foi feita a codificação, categorização e quantificação da informação, a partir de técnicas como a transcrição e leitura das entrevistas, construção das sinopses, e análise descritiva por meio de construção de tipologias e análise categorial.

A última fase foi a de tratamento dos resultados, de forma quantitativa, utilizando o software Léxica Survey (Sphinx Brasil - versão avaliação) e como apoio para validação dos dados coletados, sem excluir a interpretação qualitativa. O software importa as perguntas e respostas obtidas com as entrevistas, facilitando o tratamento dos dados e a geração de gráficos. Todos os dados obtidos são apresentados no item 3.

A aplicação do método deu-se dentro do Presídio Masculino de Florianópolis/SC, em sala cedida pelo diretor do Presídio, durante uma manhã. Foi feito um processo simplificado, com 15 entrevistas (mínimo descrito por BARDIN, 2009), para análise comparativa. O critério de seleção foi uma amostra mista, com os sujeitos que estavam de plantão naquele momento. O projeto em questão contemplou os procedimentos descritos pelo Conselho Nacional de Saúde, sob Resolução 196-1996 (BRASIL, 1996), atendendo às exigências éticas e científicas fundamentais. Para tanto, foi aplicado um TCLE - Termo de Consentimento Livre e Esclarecido, o sujeito preencheu um protocolo com informações pessoais básicas e foi utilizado outro protocolo, sem identificação do sujeito, para coleta dos dados.

Os agentes penitenciários eram chamados um a um para a entrevista na sala, onde havia duas cadeiras e uma mesa. A entrevista foi gravada, para posterior transcrição, em um Ipod Nano $5^{a}$ geração, que foi colocado entre o entrevistador e o entrevistado. Foram feitas anotações em uma folha de papel sobre as atitudes dos entrevistas face as perguntas feitas. As perguntas feitas versavam sobre o que acham de seu trabalho, como consideravam seu relacionamento com companheiros de trabalho e detentos, problemas de saúde que haviam e medicamentos que tomavam. Esta pesquisa não recebeu financiamento para sua realização.

Este estudo foi aprovado pelo Comitê de Ética em Pesquisa da Secretária da Saúde do Estado de Santa Catarina (CAAE: 38234114.0.0000.0115).

\section{RESULTADOS E DISCUSSÃO}

Foram entrevistados 10 agentes do sexo masculino e 5 agentes do sexo feminino, todos funcionários regulares do Presídio Masculino de Florianópolis/SC (tabela 1). É importante salientar que o Presídio conta com 38 agentes do sexo masculino e 20 do sexo feminino, o que torna a amostra homogênea. Os agentes penitenciários entrevistados tinham entre 28 e 61 anos, havendo uma grande variação de idade, portanto foi feita uma divisão por faixa etária sendo que $33,3 \%$ tem entre 25 e 35 anos, outros $33,3 \%$ entre 35 e 45 anos, $20 \%$ entre 45 e 55 anos e $13,3 \%$ entre 5 e 65 anos ( $\sigma=1,06)$.

A jornada de trabalho adotada por $80 \%$ dos agentes entrevistados é de $48 \mathrm{~h} / \mathrm{semanais,}$ chamada por eles de jornada " $24 \times 72$ ", onde se trabalha 24 horas seguidas e pausa 72 horas. Os outros $20 \%$ trabalhavam todos os dias, chegando a 10 horas por dia, por serem 
agentes penitenciários remanejados a área administrativa. O tempo na empresa foi também de grande variação, entre 4 e 41 anos, sendo que uma divisão por faixa de anos foi feita, resultando em 33,3\% que trabalham de 4 a 6 anos na instituição, $40 \%$ de 7 a 9 anos, $13,3 \%$ de 10 a 12 anos e outros $13,3 \%$ a mais de 20 anos ( $\sigma=1,03)$.

Tabela 1 - Descrição das características pessoais e socio-demográficas da amostra $(n=15)$ e respostas categorizadas das entrevistas.

\begin{tabular}{|c|c|c|c|}
\hline \multicolumn{4}{|c|}{ Características pessoais e socio-demográficas } \\
\hline Variáveis independentes & $\mathrm{n}(\%)$ & Variáveis independentes & $\mathrm{n}(\%)$ \\
\hline Sexo & & Estado civil & \\
\hline masculino & $10(66.7)$ & solteiro(a) & $6(40.0)$ \\
\hline feminino & $5(33.3)$ & casado(a) & $8(60.0)$ \\
\hline Idade & & Educação & \\
\hline 25 a 35 anos & $5(33.3)$ & ensino médio & $10(66.7)$ \\
\hline 35 a 45 anos & $5(33.3)$ & ensino superior & $5(33.3)$ \\
\hline 45 a 55 anos & $3(20.0)$ & & \\
\hline 55 a 65 anos & $2(13.3)$ & & \\
\hline Tempo de serviço & & Jornada de trabalho & \\
\hline 4 a 6 anos & $5(33.3)$ & $48 \mathrm{~h} / \mathrm{s}(24 \times 72)$ & $12(80.0)$ \\
\hline 7 a 9 anos & $6(40.0)$ & $>8 \mathrm{~h} / \mathrm{d}$ & $3(20.0)$ \\
\hline 10 a 12 anos & $2(13.3)$ & & \\
\hline$>20$ anos & $2(13.3)$ & & \\
\hline
\end{tabular}

A partir da gravação das 15 entrevistas, foi possível categorizar e organizar as informações obtidas (Tabela 2).

Tabela 2 - Respostas categorizadas das entrevistas ( $n=15)$.

\begin{tabular}{|c|c|c|c|}
\hline \multicolumn{4}{|c|}{ Respostas categorizadas da entrevista sobre trabalho e saúde } \\
\hline Variáveis independentes & $\mathrm{n}(\%)$ & Variáveis independentes & $\mathrm{n}(\%)$ \\
\hline $\begin{array}{l}\text { Percepção sobre trabalho } \\
\text { lembra o que vê na cadeia } \\
\text { do portão pra fora esquece } \\
\text { gosta do que faz } \\
\text { se conforma } \\
\text { afeta o psicologico } \\
\text { ficou desconfiado } \\
\text { estressante }\end{array}$ & $\begin{array}{l}2(13.3) \\
1(6.7) \\
1(6.7) \\
2(13.3) \\
10(66.7) \\
3(20.0) \\
3(20.0)\end{array}$ & $\begin{array}{l}\text { Percepção da segurança } \\
\text { medo diariamente } \\
\text { acha perigoso } \\
\text { tem mania de perseguição } \\
\text { medo de ataque de detentos }\end{array}$ & $\begin{array}{l}2(13.3) \\
6(40.0) \\
1(6.7) \\
2(13.3)\end{array}$ \\
\hline $\begin{array}{l}\text { Problemas de saúde } \\
\text { distúrbio de sono } \\
\text { tuberculose } \\
\text { rinite alérgica } \\
\text { irritabilidade }\end{array}$ & $\begin{array}{l}4(26.7) \\
2(13.3) \\
1(6.7) \\
1(6.7)\end{array}$ & $\begin{array}{l}\text { Medicamentos } \\
\text { dor de cabeça } \\
\text { ansiedade } \\
\text { respiração } \\
\text { sono } \\
\end{array}$ & $\begin{array}{l}3(20) \\
2(10) \\
2(10) \\
3(20)\end{array}$ \\
\hline
\end{tabular}




$\begin{array}{lll}\text { stress } & 6(40.0) & \text { pressão alta } \\ \text { coluna } & 2(13.3) & \\ \text { câncer } & 1(6.7) & \\ \text { pressão alta } & 1(6.7) & \\ \text { enxaqueca } & 1(6.7) & \end{array}$

Obs: a quantidade de citações pode ser superior a quantidade de observações devido à respostas múltiplas.

Quando perguntados sobre o que achavam de seu trabalho, $66,7 \%$ comentou que afetava seu psicológico, além de 13,3\% afirmar que ficava lembrando o que via na cadeia em seu tempo de folga. $13,3 \%$ também afirmaram achar o trabalho estressante e outros $13,3 \%$ afirmaram terem ficado mais desconfiados depois de começar a trabalhar como agente, como ilustra o comentário a seguir:

"é humanamente impossível você não se deixar afetar e reflete, reflete, muda totalmente a tua vida lá fora, você fica mais desconfiada, muda bastante" (E.13).

Apenas $6,7 \%$ gosta do que faz, e outros $6,7 \%$ comentaram esquecer completamente do trabalho "do portão pra fora". 13,3\% disseram se conformar com o trabalho por ser funcionário público, como apresentado a seguir:

"é uma coisa que ninguém sonha "ah, eu quero ser agente penitenciário" né?" (E.1); "mas é aquela coisa, tu é funcionário público" (E.9).

Comparando a percepção sobre o trabalho com o sexo dos agentes entrevistados, não existem muitas diferenças, entretanto as mulheres parecem mais conformadas com o serviço por serem funcionárias públicas, apesar de gostarem menos do serviço e ficarem mais desconfiadas (tabela 3 ).

Tabela 3 - Comparação entre sexo e percepção sobre o trabalho.

\begin{tabular}{|l|l|l|l|}
\hline & $\begin{array}{l}\text { Masculino } \\
\mathrm{n}(\%)\end{array}$ & $\begin{array}{l}\text { Feminino } \\
\mathrm{n}(\%)\end{array}$ & $\begin{array}{l}\text { Total } \\
\mathrm{n}(\%)\end{array}$ \\
\hline Fica lembrando o que vê na cadeia & $1(50.0)$ & $1(50.0)$ & $2(100.0)$ \\
\hline Do portão pra fora esquece completamente & $1(100.0)$ & $0(0.0)$ & $1(100.0)$ \\
\hline Gosta do que faz & $1(100.0)$ & $0(0.0)$ & $1(100.0)$ \\
\hline Se conforma por ser funcionário público & $0(0.0)$ & $2(100.0)$ & $2(100.0)$ \\
\hline Acredita que afeta o psicológico & $5(50.0)$ & $5(50.0)$ & $10(100.0)$ \\
\hline Ficou mais desconfiado & $1(33.3)$ & $2(66.7)$ & $3(100.0)$ \\
\hline Acha estressante & $2(66.7)$ & $1(33.3)$ & $3(100.0)$ \\
\hline
\end{tabular}

Com relação a segurança, questão que não foi abordada diretamente como pergunta, mas comentada por todos os entrevistados, $40 \%$ acha o trabalho perigoso, sendo que $18 \%$ comentaram ficar com mania de perseguição e outros $13,3 \%$ ter medo de ataque de detentos, e 13,3\% afirmaram lidar com o medo diariamente. Além disso, foi comentado por $33,3 \%$ dos entrevistados que não lhes é fornecido equipamentos de segurança, como armas, coletes etc.

"aqui a gente tem contato direto sem proteção nenhuma" (E.3);

"você fica marcada por uma vida inteira, você sempre vai ser uma agente penitenciaria” (E.10).

Comparando a idade com a percepção de segurança (tabela 4), pode-se perceber que as faixas etárias mais velhas (45 a 55 anos, 55 a 65 anos) não citaram problemas com 
segurança, assim como o tempo de serviço dos agentes penitenciários influenciou na sua percepção sobre segurança (tabela 5), nota-se que os mais novos na empresa (4 a 6 anos) veêm mais problemas de segurança que os outros funcionários.

Tabela 4 - Comparação entre faixa etária e percepção de segurança.

\begin{tabular}{|l|l|l|l|l|l|}
\hline & $\begin{array}{l}25 \text { a 35 anos } \\
\mathrm{n}(\%)\end{array}$ & $\begin{array}{l}35 \text { a } 45 \text { anos } \\
\mathrm{n}(\%)\end{array}$ & $\begin{array}{l}45 \text { a } 55 \text { anos } \\
\mathrm{n}(\%)\end{array}$ & $\begin{array}{l}55 \text { a } 65 \text { anos } \\
\mathrm{n}(\%)\end{array}$ & $\begin{array}{l}\text { Total } \\
\mathrm{n}(\%)\end{array}$ \\
\hline $\begin{array}{l}\text { Lida com o medo } \\
\text { diariamento }\end{array}$ & $0(0.0)$ & $2(100.0)$ & $0(0.0)$ & $0(0.0)$ & $2(100.0)$ \\
\hline Acha perigoso de & $3(50.0)$ & $3(50.0)$ & $0(0.0)$ & $0(0.0)$ & $6(100.0)$ \\
\hline $\begin{array}{l}\text { Tem mania } \\
\text { perseguição }\end{array}$ & $0(0.0)$ & $1(100.0)$ & $0(0.0)$ & $0(0.0)$ & $1(100.0)$ \\
\hline $\begin{array}{l}\text { Tem medo de ataque } \\
\text { de detentos }\end{array}$ & $1(50.0)$ & $1(50.0)$ & $0(0.0)$ & $0(0.0)$ & $2(100.0)$ \\
\hline
\end{tabular}

Tabela 5 - Comparação entre tempo de serviço e percepção de segurança.

\begin{tabular}{|l|l|l|l|l|l|}
\hline & $\begin{array}{l}4 \text { a } 6 \text { anos } \\
\mathrm{n}(\%)\end{array}$ & $\begin{array}{l}7 \text { a 9 anos } \\
\mathrm{n}(\%)\end{array}$ & $\begin{array}{l}10 \text { a 12 anos } \\
\mathrm{n}(\%)\end{array}$ & $\begin{array}{l}+20 \text { anos } \\
\mathrm{n}(\%)\end{array}$ & $\begin{array}{l}\text { Total } \\
\mathrm{n}(\%)\end{array}$ \\
\hline Lida com o medo diariamento & $2(100.0)$ & $0(0.0)$ & $0(0.0)$ & $0(0.0)$ & $2(100.0)$ \\
\hline Acha perigoso & $4(66.7)$ & $1(16.7)$ & $1(16.7)$ & $0(0.0)$ & $6(100.0)$ \\
\hline Tem mania de perseguição & $1(100.0)$ & $0(0.0)$ & $0(0.0)$ & $0(0.0)$ & $1(100.0)$ \\
\hline $\begin{array}{l}\text { Tem medo de ataque de } \\
\text { detentos }\end{array}$ & $2(100.0)$ & $0(0.0)$ & $0(0.0)$ & $0(0.0)$ & $2(100.0)$ \\
\hline
\end{tabular}

Já as palavras ou expressões mais ditas quando perguntados sobre os detentos e relacionamento com os colegas, pode ser verificada na "constelação de atributos" a seguir (figura 1). Apesar de 6 entrevistados comentarem que o relacionamento com os colegas de trabalho é bom, foram ditas também palavras negativas como brigas, desconfiança e stress, como ilustram os seguintes comentários:

"as vezes mais complica entre a gente do que até com o preso, justamente por estar em um ambiente negativo assim" (E.3);

"é mais assim, tô observando, vou me cuidar com ele, será que é ele, que ta entrando celular por ele" (E.7).

Sobre os detentos, foi comentado que:

"o relacionamento com detento é quase como se fosse com criança. Todo dia tu fala que ele não pode fazer, e todo dia ele vai tentar fazer, entendeu?" (E.4);

"não é bom que a gente saiba nenhum tipo de crime que o detento cometeu, pra que não cause um certo mal estar" (E.10). 
Figura 1 - Constelação de atributos sobre os colegas de trabalho e os detentos.
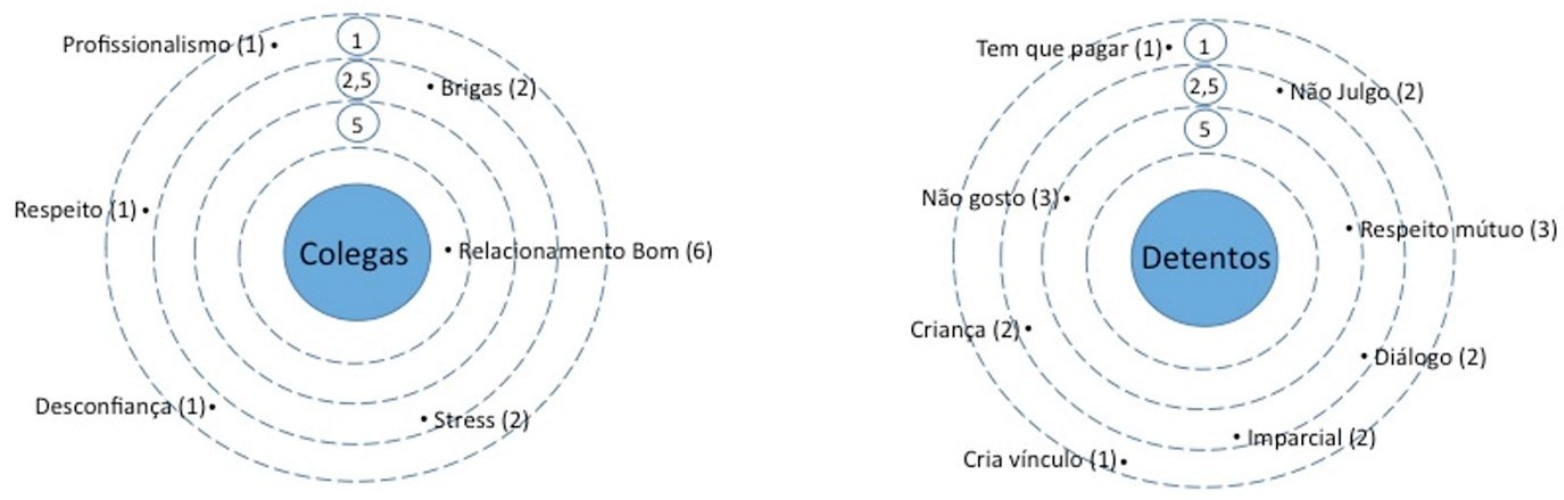

Com relação à saúde, $26,7 \%$ comentaram ter algum distúrbio de sono, já tratado ou não:

"não conseguia mais dormir direito a noite, tinha pesadelo, fiquei completamente perturbado" (E.8);

"a uns anos atrás eu precisei de um profissional psiquiatra, não dormia mais a três dias" (E.7).

$13,3 \%$ já haviam contraído tuberculose no presídio e $40 \%$ comentaram ter problemas com stress, assim como outros trabalhos realizados em presídios brasileiros (Gomes, 2009 e Santos et al., 2010). Apesar de não relatarem problemas psicológicos específicos, a frase a seguir ilustra que os problemas existem:

"psicológico assim muitos colegas vão te dizer que não tem, mas, se eles forem sinceros vão te dizer que tem" (E.3).

Foram relatados também casos de rinite alérgica, irritabilidade, dores na coluna lombar, pressão alta e enxaqueca em menor quantidade $(6,7 \%$ cada).

$66,6 \%$ dos entrevistados afirmaram tomar medicamentos frequentemente, sendo que $20 \%$ tomam remédio para dor de cabeça, $10 \%$ para ansiedade, outros $10 \%$ para respiração, $20 \%$ para problemas de sono e $10 \%$ para problemas de pressão alta. Comparando os problemas de saúde relatados com a idade dos agentes entrevistados, pode-se perceber que o distúrbio do sono é constante em todas as faixas etárias, entretanto o stress e problemas de irritabilidade parece ser mais comum nos agentes mais jovens, entre 25 e 45 anos (tabela $6)$.

Tabela 6 - Comparação entre faixa etária e problemas de saúde.

\begin{tabular}{|l|l|l|l|l|l|}
\hline & $\begin{array}{l}25 \text { a 35 anos } \\
\mathrm{n}(\%)\end{array}$ & $\begin{array}{l}\text { 35 a 45 anos } \\
\mathrm{n}(\%)\end{array}$ & $\begin{array}{l}45 \text { a 55 anos } \\
\mathrm{n}(\%)\end{array}$ & $\begin{array}{l}55 \text { a 65 anos } \\
\mathrm{n}(\%)\end{array}$ & $\begin{array}{l}\text { Total } \\
\mathrm{n}(\%)\end{array}$ \\
\hline Distúrbio do sono & $1(25.0)$ & $1(25.0)$ & $1(25.0)$ & $1(25.0)$ & $4(100.0)$ \\
\hline Tuberculose & $1(50.0)$ & $0(0.0)$ & $1(50.0)$ & $0(0.0)$ & $2(100.0)$ \\
\hline Rinite alérgica & $1(100.0)$ & $0(0.0)$ & $0(0.0)$ & $0(0.0)$ & $1(100.0)$ \\
\hline Irritabilidade & $1(100.0)$ & $0(0.0)$ & $0(0.0)$ & $0(0.0)$ & $1(100.0)$ \\
\hline Stress & $2(33.3)$ & $2(33.3)$ & $1(16.7)$ & $1(16.7)$ & $6(100.0)$ \\
\hline Coluna & $0(0.0)$ & $1(50.0)$ & $1(50.0)$ & $0(0.0)$ & $1(100.0)$ \\
\hline Câncer & $0(0.0)$ & $0(0.0)$ & $1(100.0)$ & $0(0.0)$ & $1(100.0)$ \\
\hline Pressão alta & $0(0.0)$ & $0(0.0)$ & $1(100.0)$ & $0(0.0)$ & $1(100.0)$ \\
\hline Enxaqueca & $0(0.0)$ & $0(0.0)$ & $1(100.0)$ & $0(0.0)$ & $1(100.0)$ \\
\hline
\end{tabular}


Pode-se perceber pela abordagem de campo realizada que o conteúdo do trabalho afeta o psicológico dos agentes, por ser caracterizado por um alto nível de exigências psicológicas (JOHNSON et al., 2005; GHADDAR et al., 2008), além de contribuir para elevar seu nível de estresse, como posto por Finn (1998), Huckabee (1992), Schaufeli e Peeters (2000) pela alta tensão envolvida neste tipo de trabalho. A falta de estrutura e equipamentos de segurança, faz com que os agentes fiquem inseguros e lidem com o medo diariamente, fato também identificado por Santos (2010) e Armstrong e Griffin (2004). Entretanto, com o decorrer dos anos, essa insegurança parece desaparecer, possivelmente pelo hábito com o trabalho:

"se existe o perigo mesmo, a gente fica mais habituado com ele" (E.4).

A situação de trabalho faz com que os agentes tenham mais contato com crimes do que outros tipos de profissões, e segundo Armstrong e Griffin (2004), percebem uma ameaça constante de perigo, o que os torna mais desconfiados:

"você percebe que qualquer um tá sujeito a qualquer coisa, e que essas coisas acontecem sempre (...) dai você fica sempre mais desconfiada, mais protetora, porque eu tenho 2 filhos né" (E.13).

O relacionamento com os colegas de trabalho é bom, ainda que existam brigas pela pressão que o trabalho acarreta. Apesar dos detentos serem vistos como pessoas que sempre tentarão fazer algo errado, existe respeito mútuo e um bom diálogo dentro do presídio, diferentemente dos resultados encontrados em estudos em penitenciárias do Rio de Janeiro (VASCONCELOS, 2000), que afirmam que é permeado pelo fenômeno da violência. Essa diferença possivelmente se dá pela diferente forma de tratamento dos presos em presídios e penitenciárias, onde nesta última o tratamento é mais rígido e com maior confinamento.

A falta de higiene e ventilação, além da alta umidade dentro do presídio são prejudiciais à saúde, podendo provocar diversas doenças, como tuberculose e rinite alérgica, identificados também em penitenciárias do Rio de Janeiro, de Salvador e de São Paulo (VASCONCELOS, 2000; FERNANDES et al., 2002; RUMIN, 2006). Os distúrbios de sono, problemas de irritabilidade e até pressão alta e dores na coluna podem ser relacionados ao conteúdo do trabalho e nível de estresse do trabalhador, também descritos como alguns dos efeitos do estresse nos agentes penitenciários nos estudos de Anson et al. (1997) e DeCarlo e GruenFeld (1989).

\section{CONSIDERAÇÕES FINAIS}

Os resultados apresentados através da análise da percepção do trabalho e saúde pelos agentes penitenciários evidenciam que o tipo de trabalho e seu conteúdo elevam o nível de stress dos trabalhadores analisados, sendo que $40 \%$ apresentam problemas de stress. A falta de segurança (40\% acham o trabalho perigoso) gera medo $(13,3 \%)$ e desconfiança $(20 \%)$, revelando problemas na organização e no processo de trabalho.

Com a análise de conteúdo foi possível verificar a percepção dos agentes sobre seu trabalho e saúde, entretanto, uma análise mais profunda da condição de trabalho é fundamental para compreender o quanto o contexto de trabalho influencia em sua saúde. Este estudo de caso não analisa profundamente os aspectos do trabalho em sistemas prisionais, entretanto, espera-se que este estudo tenha evidenciado a relevância da interrelação entre o trabalho e a saúde do trabalhador, permitindo a análise dos aspectos do trabalho que trazem riscos de adoecimento. 


\section{REFERÊNCIAS BIBLIOGRÁFICAS}

ANSON, R. H.; JOHNSON, B.; ANSON, N. W. Magnitude and source of general and occupationspecific stress among police and correctional officers. Journal of Offender Rehabilitation, v.25, p.103 - 113, 1997.

ARMSTRONG, G. S.; GRIFFIN, M. Does the job matter? Comparing correlates of stress among treatment and correctional staff in prisons. Journal of Criminal Justice, v.32, p.577-592, 2004.

BABAZONO, A; MINO, Y; NAGANO, J; TSUDA, T; ARAKI, T. A prospective study on the influences of workplace stress on mental health. Journal of Occupational Health, v.47, p.490-495, 2005.

BARDIN, L. Análise de Conteúdo. Lisboa, Portugal: Edições 70, LDA, 2009.

BOURBONNAIS, R.; JAUVIN, N.; DUSSAULT, J.; VÉZINA, M. Psychosocial work environment, interpersonal violence at work and mental health among correctional officers. International Journal of Law and Psychiatry, v.30, p.355-368, 2007.

BRASIL - CONSELHO NACIONAL DE SAÚDE. Resolução No 196, de 10 de outubro de 1996. Disponível em: http://conselho.saude.gov.br/docs/Resolucoes/Reso196.doc. Acesso em: 20 set. 2014.

CAMP, S.; LAMBERT, E. The influence of organizational incetives on absenteeism: sick-leave use among correctional workers. Criminal Justice Policy Review, v.17, p.144-172, 2006.

CASSITO, M; FATTORINI, E; GILIOLO, R; RENGO, C. Raising awareness to psychological harassment at work- Protecting Workers' Health series. Milano: World Health Organization, 2003, p.16-23.

CHEEK, F. E. Stress management for correctional officers and their families. College Park, MD: American Correctional Association, 1984.

CHENG, Y; KAWACHI, I; COAKLEY, E; SCHWARTS, J; COLDITZ, G. Association between psychosocial work characteristics and health functioning in American women: prospective study. British Medical Journal, v.320, p.1432-1436, 2000.

DAMAS, F. B. Assistência e condições de saúde nas prisões de Santa Catarina, Brasil. Revista de Saúde Pública de Santa Catarina, n. 5, v. 3, p. 6-22, 2012.

DIUANA, V.; LHUILIER, D.; SÁNCHEZ, A. R.; AMADO, G.; ARAÚJO, L.; DUARTE, A. Saúde em prisões: representações e práticas dos agentes de segurança penitenciária no Rio de Janeiro, Brasil. Cadernos de Saúde Pública, n. 24, v.8, p. 1887-1896, 2008.

DE CARLO, D. T.; GRUENFELD, D. H. Stress in the American workplace: Alternatives for the working wounded. Fort Washington, PA7 LRP Publications, 1989.

DEJOURS, C. A loucura do trabalho: estudo de psicopatologia do trabalho. São Paulo: Cortez Oboré, 1992.

FERNANDES, R.; SILVANY NETO, A.; SENA, G.; LEAL, A. CARNEIRO, C; COSTA, F. Trabalho e cárcere: um estudo com agentes penitenciários da Região Metropolitana de Salvador, Brasil. Cadernos de Saúde Pública, v.18, p.807-816, 2002.

FINN, P. Correctional officer stress: A cause for concern and additional help. Federal Probation, v.62, p.65-74, 1998.

GHADDAR, A.; MATEO, I.; SANCHEZ, P. Occupational stress nad mental health among officers: a cross-sectional study. Journal of Occupational Health, v.50, p.92-98, 2008.

GIL, A. C. Como elaborar projetos de pesquisa. 3 ed. São Paulo: Atlas, 1996. 
GOLDBERG, P.; DAVID, S.; LANDRE, M.; GOLDBERG, M.; DASSA, S.; FUHRER, R. Work conditions and mental health among prison staff in France. Scandinavian Journal of Work, Environment \& Health, v.22, n.1, p. 45-54, 1996.

GOMES, S. M. Sofrimento mental e satisfação no trabalho em professores de unidades prisionais em Porto Velho. Brasília, 2009. Dissertação (Mestrado em Ciências da Saúde) Programa de Pós-Graduação em Ciências da Saúde, Universidade de Brasília, 2009.

HIGASHIGUCHI K, NAKAGAWA H, MORIKAWA, Y; ISHIZAKI, M; MIURA, K; NARUSE, Y; KIDO, T. The association between job demand, control and depression in workplaces in Japan. Journal of Occupational Health, v.44, p.427-428, 2002.

HUCKABEE, R. G. Stress in corrections: An over- view of the issues. Journal of Criminal Justice, v.20, p.479-486, 1992.

ISHIZAKI, M; KAWAKAMI, N; HONDA, R; NAKAGAWA, H; MORIKAWA, Y; YAMADA Y. The Japan work stress and health cohort study group: psychosocial Word characteristics and sickness absence in Japanese employees. International Archives of Occupational and Environmental Health, v.7, p.640-646, 2006.

JOHNSON, S.; COOPER, C.; CARTWRIGHT, S.; DONALD, I.; TAYLOR, P.; MILLET, C. The experience of work relates stress across occupations. Journal of Managerial Psychology, v.20, p.12,2005

LIU, L.; HU, S.; WANG, L.; SUI, L.; MA, L. positive resources for combating depressive symptoms among Chinese male correctional officers: perceived organizational support and psychological capital. BMC Psychiatry, v.13, p.89, 2013.

OBIDOA, C.; REEVES, D.; WARREN, N.; REISINE, S.; CHERNIACK, M. Depression and work family conflict among corrections officers. Journal of Occupational and Environmental Medicine, v.53, n.11, p.1294-1301, 2011.

ORGANIZAÇÃO INTERNACIONAL DO TRABALHO (OIT). Relatório Global do Seguimento da Declaração da OIT relativa a Princípios e Direitos Fundamentais no Trabalho. Disponível em: http://www.oit.org.br/sites/all/forced_labour/oit/relatorio/relat_global.pdf. Acesso em: 30 abr. 2014.

REINERT, F.; MERINO, E.C.D.; GONTIJO, L.A. Análise das condições de trabalho do assistente social no Presídio Feminino de Florianópolis/SC. Ação Ergonomica, v.9, n.2, p.97-106, 2014.a

RUMIN, C. Sofrimento e vigilância prisional: o trabalho e a atenção em saúde mental. Psicologia: ciência e profissão, v.26, n.4, p.570-581, 2006.

SANTOS, D.; DIAS, J.; PEREIRA, M.; MOREIRA, T.; BARROS, D.; SERAFIM, A. Prevalência de transtornos mentais comuns em agentes penitenciários. Revista Brasileira de Medicina do Trabalho, v.8, n.1, p.33-38, 2010.

SANTOS, M. Agente penitenciário: trabalho no cárcere. Natal, 2010. Dissertação (Mestrado em Psicologia) - Programa de Pós-Graduação em Psicologia, Universidade Federal do Rio Grande do Norte, 2010.

SCHAUFELI, W. B.; PEETERS, M. C. W. Job stress and burnout among correctional officers: A literature review. International Journal of Stress Management, v.7, p.19-48, 2000.

SENOL-DURAK, E.; DURAK, M.; GENCOZ, T. Development of work stress scale for correctional officers. Journal of Occupational Rehabilitation, v.16, n.1, p.157-168, 2006.

STANSFELD, S; CANDY, B. Psychological work environment and mental health - a meta-analysis review. Scandinavian Journal of Work, Environment \& Health, v.32, p.443-462, 2006. 
SUI, G.; HU, S.; SUN, W.; WANG, Y.; LIU, L.; YANG, X.; WANG, L. Prevalence and associated factors of depressive symptoms among Chinese male correctional officers. International Archives of Occupational and Environmental Health, v.87, p.387-395, 2014.

TARTAGLINI, A.; SAFRAN, D. A topography of psychiatric disorders among correction officers. Journal of Occupational and Environmental Medicine, v. 39, p. 569-573, 1997.

TRIVIÑOS, A. N. S. Introdução à pesquisa em ciências sociais: a pesquisa qualitativa em educação. São Paulo: Atlas, 2006.

TSUTSUMI, A.; KAYABA, K.; NAGAMI, M.; MIKI, A.; KAWANO, Y.; OHYA, Y.; ODAGIRI, Y.; SHIMOMITSU. The effort-reward imbalance model: experience in Japaes working population. Journal of Occupational Health, v.44, p.398-407, 2002.

VASCONCELOS, A. A saúde sob custódia: um estudo sobre agentes de segurança penitenciária no Rio de Janeiro. Rio de Janeiro, 2000. Dissertação (Mestrado em Saúde Pública) Centro de estudos da saúde do trabalhador e ecologia humana, Escola Nacional de Saúde, 2000.

WOODRUFF, L. Occupational stress for correctional personnel. American Jails, v. 7, p. 15-20, 1993. 